\title{
Doença Invasiva Pneumocócica Pediátrica Antes da Vacinação Universal: 1995 - 2015
}

\author{
Paediatric Invasive Pneumococcal Disease Before \\ Universal Vaccination: 1995 - 2015
}

Muriel FERREIRA ${ }^{1}$, Henrique OLIVEIRA ${ }^{2}$, Nuno Costa e SILVA ${ }^{2}$, Luís JANUÁRIO ${ }^{1}$, Fernanda RODRIGUES 1,3 Acta Med Port 2017 Jun;30(6):457-462 - https://doi.org/10.20344/amp.8493

\begin{abstract}
RESUMO
Introdução: A vacina conjugada pneumocócica foi introduzida no mercado privado português em 2001, atingindo ao longo dos anos coberturas moderadamente elevadas. Em julho de 2015 foi integrada no Programa Nacional de Vacinação. O objetivo deste estudo foi caracterizar a doença invasiva pneumocócica num hospital pediátrico antes da vacinação universal.

Material e Métodos: Análise retrospetiva dos processos clínicos de todas as crianças com identificação de Streptococcus pneumoniae por cultura e/ou por biologia molecular (disponível desde 2008), em produtos obtidos de locais estéreis, de janeiro 1995 a junho 2015 , avaliando dados demográficos, clínicos e microbiológicos. Os serotipos estão disponíveis desde 2004.

Resultados: Ao longo destes 20 anos identificámos 112 casos de doença invasiva pneumocócica, com idade mediana de 15 meses ( 1 mês - 15 anos). A mediana de casos/ano foi 4, com valores máximos entre 2001 - 2002 (8/ano) e 2007 - 2012 (7 - $11 / a n o)$. A identificação ocorreu maioritariamente em hemocultura (72), líquido cefalorraquidiano (24), líquido pleural (11) e outros (5). Os diagnósticos mais frequentes foram pneumonia (38\%), bacteriemia oculta $(34 \%)$ e meningite $(21 \%)$. Ao longo do período em análise, observou-se um aumento do diagnóstico de pneumonia e aumento ligeiro de bacteriemia oculta, tendo-se mantido relativamente constante o de meningite.
\end{abstract}

Discussão: Nas últimas duas décadas não se observou redução do número de casos de doença invasiva pneumocócica, tendo ocorrido um aumento da identificação de pneumococo em pneumonia e bacteriemia oculta, para o qual poderão ter contribuído a introdução dos métodos de biologia molecular e a realização de mais hemoculturas. Os serotipos vacinais foram predominantes.

Conclusão: Esta analise retrospetiva pré vacinação universal, contribuirá para avaliar o impacto da vacinação na população pediátrica portuguesa.

Palavras-chave: Criança; Infecções Pneumocócicas; Portugal; Vacinas Conjugadas; Vacinas Pneumocócicas

\section{ABSTRACT}

Introduction: Pneumococcal conjugate vaccine was introduced in the private market in Portugal in 2001, reaching over the years a moderately high coverage. In July 2015, it was included in the National Immunisation Program. The aim of this study was to characterize invasive pneumococcal disease in a pediatric hospital before universal use of the vaccine.

Material and Methods: Retrospective analysis of medical records of all children with Streptococcus pneumoniae identified by culture and/or molecular biology (available since 2008), in products obtained from sterile sites, from January 1995 to June 2015. We evaluated demographic, clinical and microbiological data. Serotype results are available since 2004.

Results: Over those 20 years, 112 invasive pneumococcal disease cases were identified, with a median age of 15 months (1 month - 15 years). The median number of cases /year was 4, the highest between 2001 - 2002 (8/year) and 2007 - 2012 (7 - 11/year). The identification occurred mostly in blood culture (72), cerebrospinal fluid (24), pleural fluid (11) an others (5). The most frequent diagnoses were pneumonia $(38 \%)$, occult bacteraemia $(34 \%)$ and meningitis $(21 \%)$. Over the period under review, there was an increase of pneumonia and slight increase of $\mathrm{OB}$, with meningitis cases remaining relatively unchanged.

Discussion: In the last two decades, there was no reduction in the number of cases of invasive pneumococcal disease. There was an increase in isolates from pneumonia and occult bacteraemia that might be due to the introduction of molecular biological methods for Streptococcus pneumoniae detection. Vaccine serotypes were predominant.

Conclusion: This retrospective analysis before universal vaccination will contribute to evaluate the impact of vaccination in the Portuguese pediatric population.

Keywords: Child; Pneumococcal Infections; Pneumococcal Vaccines; Portugal; Vaccines, Conjugate

\section{INTRODUÇÃO}

Streptococcus pneumoniae (S. pneumoniae) é uma bactéria Gram positiva, que pode causar um amplo espectro de doenças na criança e adolescente, desde infeções comuns como otite média aguda e sinusite, até infeções menos frequentes e potencialmente graves como pneumonia, bacteriemia oculta (BO), sépsis e meningite. ${ }^{1}$ Após introdução da vacinação universal contra Haemophilus influenza tipo b e meningococco do grupo $\mathrm{C}$, o pneumoco- co tornou-se, nalguns países, a causa mais frequente de doença invasiva bacteriana em crianças com idade inferior a dois anos. ${ }^{2}$

Coloniza a nasofaringe humana, sendo esta a fonte de transmissão da bactéria a outros indivíduos e o ponto de partida da doença. Contudo, a maioria dos portadores não desenvolverá a doença. Em estudos realizados em infantários de Lisboa e Coimbra, cerca de $60 \%$ das crianças estão

1. Unidade de Infeciologia e Serviço de Urgência. Hospital Pediátrico. Centro Hospitalar e Universitário de Coimbra. Coimbra. Portugal.

2. Serviço de Patologia Clínica. Centro Hospitalar e Universitário de Coimbra. Coimbra. Portugal.

3. Departamento de Pediatria. Faculdade de Medicina. Universidade de Coimbra. Coimbra. Portugal.

$\triangle$ Autor correspondente: Muriel Ferreira. murielguardaferreira@gmail.com

Recebido: 19 de novembro de 2016 - Aceite: 27 de março de 2017 | Copyright @ Ordem dos Médicos 2017 
colonizadas por esta bactéria. ${ }^{3,4}$ São conhecidos mais de 90 serotipos mas a maioria dos casos de doença na criança é causado por número relativamente pequeno. ${ }^{1}$

A primeira vacina antipneumocócica comercializada em Portugal foi a vacina polissacárida 23-valente (Pneumo23 $\left.{ }^{\circledR}\right){ }^{1,5}$ É recomendada para grupos de risco e uma avaliação recente efetuada num hospital central do norte do país mostrou uma baixa utilização nesta população. ${ }^{6}$

Em 2001, a vacina conjugada antipneumocócica 7-valente (VCP7v, Prevenar ${ }^{\circledR}$, Wyeth) foi autorizada na Farmácia Comunitária e começou a ser comercializada sem comparticipação e mediante receita médica. Em 2009 foi introduzida a vacina conjugada 10-valente (Synflorix ${ }^{\circledR}$, GSK) e em 2010, a vacina conjugada 13-valente (VCP13v, Prevenar13 ${ }^{\circledR}$, Pfizer) substituiu a 7-valente. A sua utilização tem sido recomendada pela Comissão de Vacinas da Sociedade Portuguesa de Pediatria desde 2010. ${ }^{5}$

Com a utilização das vacinas conjugadas, verificou-se redução importante dos casos da doença invasiva pneumocócica (DIP), da colonização e da doença não invasiva causada por serotipos vacinais nas crianças vacinadas e em indivíduos não vacinados (imunidade de grupo). Quase simultaneamente, assistiu-se ao aumento de DIP causada por serotipos não vacinais e aparecimento de serotipos não vacinais na nasofaringe (fenómeno de substituição), não se alterando significativamente as taxas de colonização. ${ }^{2,7,8}$

Desde 2006, a Sociedade de Infecciologia Pediátrica da Sociedade Portuguesa de Pediatria coordena um estudo prospetivo nacional de vigilância de DIP em idade pediátrica, observando-se uma redução da incidência global de DIP em Portugal (MJ Brito, comunicação pessoal). Dados laboratoriais recentemente publicados mostram que esta diminuição terá resultado essencialmente da redução significativa do número de casos causados pelos serotipos adicionais da VCP10v e VCP13v $(1,5,7 F)$, especialmente o serotipo 1 nas crianças mais velhas; e pelos serotipos adicionais da VCP13v $(3,6 \mathrm{~A}, 19 \mathrm{~A})$, particularmente o 19A em crianças mais jovens. Os serotipos incluídos na VCP13v permaneciam, no entanto, importantes causas de DIP, correspondendo a $63,2 \%$ dos casos isolados em Portugal em $2011-2012 .^{9}$

Em Portugal, em 2010, a VCP13v passou a ser disponibilizada gratuitamente aos grupos de risco, tendo sido integrada no PNV em julho de 2015.

Por este motivo, não há dados exatos sobre a evolução da cobertura vacinal mas estima-se que se aproximava de $80 \%$ em 2007 e seria de cerca de 60\% em 2014 (Brito MJ, comunicação pessoal). ${ }^{10}$ Uma avaliação do SINUS Módulo de vacinação, efetuada na Região Centro mostrou coberturas aos 24 meses para $\geq$ três doses de $69 \%$ em 2008 e 73\% em 2011 (cortesia de Viveiros D, dados não publicados).

O objetivo do estudo foi caracterizar a DIP na população de um hospital pediátrico antes da introdução da VCP13v no PNV.

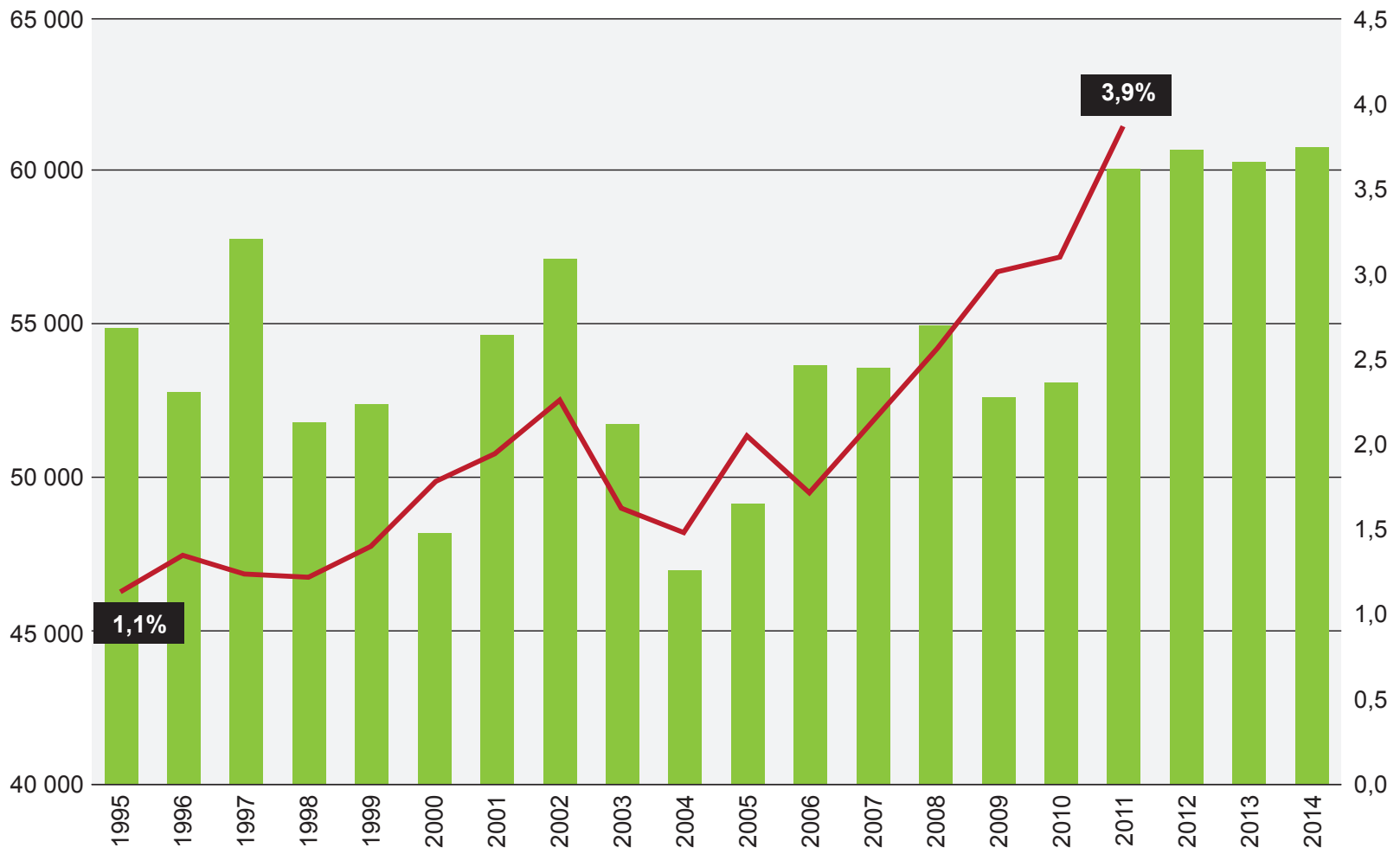




\section{MATERIAL E MÉTODOS}

Estudo retrospetivo descritivo com análise dos processos clínicos das crianças e adolescentes com diagnóstico de DIP, observadas num serviço de urgência (SU) de um hospital pediátrico, no período compreendido entre 1 de janeiro de 1995 e 30 de junho de 2015 (20,5 anos).

O SU pediátrico serve uma população de aproximadamente 389000 crianças e adolescentes da Região Centro (INE, Censos 2011). ${ }^{11}$ Funciona como centro de referência terciário, prestando também cuidados secundários e primários.

Definiu-se DIP como a identificação de S. pneumoniae num produto biológico considerado estéril (sangue, líquido cefalorraquidiano, pleural e peritoneal, pús de abcesso).

Foram analisadas variáveis demográficas clínicas, estado vacinal, dados microbiológicos, incluindo local de isolamento e método de identificação por cultura (utilizando métodos padrão em uso no Laboratório de Microbiologia) e/ou PCR, disponível desde 2008, no Instituto de Microbiologia, Faculdade de Medicina, Universidade de Lisboa. A serotipagem efetuada no Instituto de Microbiologia, Faculdade de Medicina, Universidade de Lisboa, está disponível desde 2004.

Foram também analisados os números de admissões (1995 - 2014) e de hemoculturas efetuadas no SU do hospital de 1995 a 2011 (Serviço de Estatística do CHC/CHUC e Laboratório de Microbiologia), para avaliar se houve alterações na investigação microbiológica ao longo do período em estudo.
A análise estatística foi efetuada recorrendo ao programa SPSS ${ }^{\circledR}$ for Windows, versão 22. Foi calculado o coeficiente de correlação de Pearson para avaliar a tendência evolutiva dos casos de doença. Foi considerada significância estatística se $p<0,05$.

\section{RESULTADOS}

No período analisado, assistiu-se a um aumento do número de episódios de urgência, mais evidente a partir de 2011, coincidente com a mudança para novas instalações hospitalares e alargamento da idade de atendimento até aos 18 anos, mantendo-se estes números relativamente estáveis desde então. O mesmo se verificou na percentagem de hemoculturas efetuadas relativamente ao total das admissões, assistindo-se a um aumento gradual, em particular desde 2006, tendo atingido um valor máximo de 3,9\% em 2011 (Fig.1).

Ao longo dos 20,5 anos, foram diagnosticados 112 casos de DIP, verificando-se uma tendência de aumento $(R=$ 0,$565 ; p=0,008$ ). A mediana de casos por ano foi quatro, verificando-se dois períodos com maior número: $2001 \mathrm{e}$ 2002 com oito casos/ano e 2007 a 2012 (com 8 - 11 casos/ ano) (Fig.2).

Todas as crianças e adolescentes estudados eram de raça caucasiana e verificou-se um discreto predomínio do sexo masculino (55\%).

Relativamente à distribuição etária, variou entre um mês e 15 anos, com mediana de 15 meses e média de dois anos e sete meses. Foi mais frequente entre os seis e

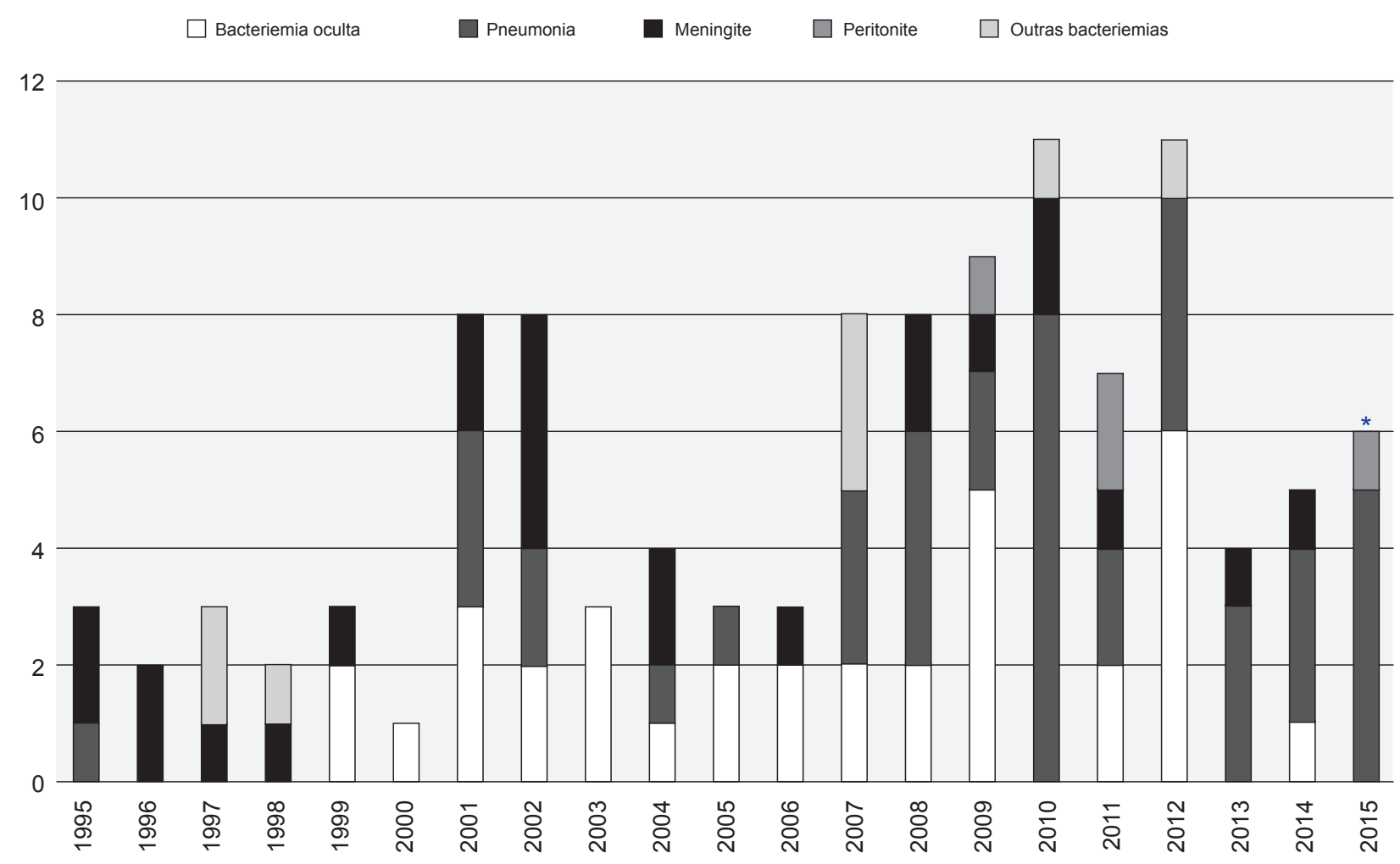

Figura 2 - Distribuição anual do número de casos e diagnósticos de doença invasiva pneumocócica * Dados até junho de 2015 
24 meses com $36,6 \%$ dos casos no primeiro ano de vida; $64,3 \%$ até aos dois anos e $85,7 \%$ até aos seis anos.

Não foi possível averiguar o estado vacinal em 19,6\% dos casos; nos restantes, $46,4 \%$ não estavam vacinados e $34 \%$ tinham recebido pelo menos uma dose de VCP. Três crianças vacinadas tiveram doença causada por um serotipo vacinal. Apenas uma tinha esquema de vacinação completo para a idade: menina de três anos com três doses de VCP13v (2, 4 e 12 meses), com pneumonia e isolamento de pneumococo serotipo 3 em hemocultura. Foi excluída imunodeficiência.

A identificação do pneumococo ocorreu em hemocultura $(n=72)$, LCR $(n=24)$, líquido pleural ( $n=11)$, pús de abcesso ( $n=3$; um abcesso retro-ocular e duas apendicites com plastron), líquido peritoneal $(n=2)$. Em seis casos, foi identificado em mais do que um produto biológico (hemocultura + LCR, $\mathrm{n}=5$; hemocultura + líquido pleural, $n=1$ ) (Tabela 1). Os diagnósticos mais frequentes foram pneumonia, bacteriemia oculta e meningite (Tabela 1). Assinalam-se na Tabela 1 os casos com identificação de $S$. pneumoniae por biologia molecular, sendo os restantes por cultura.

No decorrer do período de estudo, verificou-se um aumento dos diagnósticos de $\mathrm{BO}$ e pneumonia, tendo diminuído a meningite. A distribuição dos diagnósticos ao longo dos anos é apresentada na Fig. 2.

Analisando os diagnósticos mais frequentes, agrupados em períodos de cinco anos (Tabela 2), verificou-se um aumento do número de casos de pneumonia $(R=0,949$; $p=0,051)$, um ligeiro aumento do número de $\mathrm{BO}(\mathrm{R}=$ $0,666 ; p=0,334)$, mantendo-se o número de meningites estável ao longo do período de estudo. Neste intervalo de tempo, onze casos de pneumonia e três casos de meningite foram diagnosticados por PCR.

Estão disponíveis serotipos de $44 \%$ dos casos (49/112). Os mais frequentemente identificados foram 19A e 3 (Tabela 3).

Durante o período de estudo, registaram-se 4 óbitos, todos em contexto de meningites. Nas sequelas registadas, destacam-se surdez $(n=6)$, atraso de desenvolvimento psicomotor $(n=5)$, hidrocefalia $(n=3)$ e parésia de nervos cranianos $(n=1)$.

\section{DISCUSSÃO}

A introdução das vacinas conjugadas pneumocócicas veio modificar a epidemiologia da doença pneumocócica nas últimas décadas, com redução importante da incidência de DIP causada por serotipos vacinais nos vacinados e não vacinados. Apesar do fenómeno de substituição observado, a redução importante dos casos vacinais, ultrapassou largamente o aumento dos não vacinais. ${ }^{12-15}$

Em Portugal, a DIP tornou-se uma doença de declaração obrigatória apenas em 2014. ${ }^{17}$ Os dados do estudo prospetivo promovido pelo Grupo de Estudo da Doença Invasiva Pneumocócica da Sociedade de Infeciologia Pediátrica da Sociedade Portuguesa de Pediatria (SPP), mostram que a incidência global da doença variou entre 6,3 e 4,6/100 000 crianças e adolescentes, de 2006 a 2014 (Brito MJ, comunicação pessoal).

Há dois estudos nacionais publicados referentes à experiência de um hospital: um hospital do grupo II de Lisboa descreve uma taxa de incidência de 11,8:100 000 em 200718; outro do Norte do país descreve 32 casos de DIP ao longo de 13 anos (2000 - 2012) dificultando a análise da tendência evolutiva do da doença. ${ }^{19}$

Tabela 1 - Diagnósticos e produto biológico de identificação de S. pneumoniae

\begin{tabular}{|c|c|c|c|}
\hline Diagnósticos & n (\%) & Produto biológico de identificação (n) & Identificação por biologia molecular (n) \\
\hline Pneumonia & $42(37,5 \%)$ & $\begin{array}{l}\text { Líquido pleural (11) } \\
\text { Hemocultura (26) }\end{array}$ & Liquido pleural (11) \\
\hline Bacteriemia oculta & $38(33,9 \%)$ & Hemocultura (38) & \\
\hline Meningite & $24(21,4 \%)$ & $\begin{array}{l}\text { LCR (24) } \\
\text { Hemocultura (5) }\end{array}$ & LCR (3) \\
\hline $\begin{array}{l}\text { Apendicite } \\
\text { Peritonite primária }\end{array}$ & $4(3,6 \%)$ & $\begin{array}{l}\text { Pús abcesso (2) } \\
\text { Líquido peritoneal (2) }\end{array}$ & \\
\hline $\begin{array}{l}\text { Celulite orbitária } \\
\text { Abcesso retro ocular }\end{array}$ & $2(1,8 \%)$ & $\begin{array}{l}\text { Hemocultura (1) } \\
\text { Pús abcesso (1) }\end{array}$ & \\
\hline Sepsis & $1(0,9 \%)$ & Hemocultura (1) & \\
\hline Endocardite & $1(0,9 \%)$ & Hemocultura (1) & \\
\hline
\end{tabular}

LCR: Líquido cefalorraquidiano

Tabela 2 - Evolução temporal dos principais diagnósticos

\begin{tabular}{|c|c|c|c|c|c|}
\hline Diagnósticos (n) & 1995 - 1999 & $2000-2004$ & $2005-2009$ & $2010-2015$ & $\begin{array}{l}R \\
p\end{array}$ \\
\hline Bacteriemia oculta & 2 & 10 & 13 & 9 & $\begin{array}{l}\mathrm{R}=0,666 \\
p=0,334\end{array}$ \\
\hline Pneumonia & 1 & 6 & 10 & 25 & $\begin{array}{l}\mathrm{R}=0,949 \\
p=0,051\end{array}$ \\
\hline Meningite & 7 & 8 & 4 & 5 & $\begin{aligned} \mathrm{R} & =-0,707 \\
p & =0,293\end{aligned}$ \\
\hline
\end{tabular}


Tabela 3 - Distribuição anual dos serotipos, agrupados em serotipos da VCP7v, serotipos adicionais da VCP13v e serotipos não vacinais

\begin{tabular}{|c|c|c|c|c|c|c|c|c|c|c|c|c|c|c|}
\hline & \multicolumn{3}{|c|}{$\begin{array}{l}\text { Serotipos contidos } \\
\text { na VCP7v }\end{array}$} & \multicolumn{4}{|c|}{$\begin{array}{c}\text { Serotipos adicionais contidos } \\
\text { na VCP13v }\end{array}$} & \multicolumn{7}{|c|}{ Serotipos não vacinais } \\
\hline & 14 & $19 F$ & $23 \mathrm{~F}$ & 1 & 3 & $7 F$ & 19A & $7 \mathrm{C}$ & $10 \mathrm{~A}$ & $15 B / C$ & $22 \mathrm{~F}$ & $24 \mathrm{~F}$ & 29 & $35 \mathrm{~F}$ \\
\hline 2004 & & & & & & 1 & & & & & & & & \\
\hline 2005 & & & & & & & & & & & & 1 & & \\
\hline 2006 & & & & & 1 & & & & & & & & & \\
\hline 2007 & & & & & 1 & & 1 & & & & & & & \\
\hline 2008 & & & & 3 & 1 & & 4 & & & & & & & \\
\hline 2009 & 1 & 1 & & & 1 & & 1 & & & 1 & & & 1 & \\
\hline 2010 & 1 & & 1 & & 4 & & 2 & & & 1 & & & & \\
\hline 2011 & & & & 1 & & 1 & 3 & & & & & & & \\
\hline 2012 & 1 & 2 & & 1 & & & 3 & & & & 1 & 1 & & \\
\hline 2013 & & & & & 1 & & & & & & & & & 1 \\
\hline \multicolumn{15}{|l|}{2014} \\
\hline 2015 & & 1 & & & 1 & & 1 & 1 & 1 & & & & & \\
\hline Total & 3 & 5 & 1 & 5 & 10 & 1 & 14 & 1 & 1 & 2 & 1 & 2 & 1 & 1 \\
\hline
\end{tabular}

O presente estudo abrange um maior número de anos analisados. Não observámos, de forma sustentada, redução do número de casos de DIP ao longo dos anos. É, no entanto, necessária cautela na interpretação e comparação de dados referentes a um longo período de tempo, para os quais poderão ter contribuído mudanças de práticas nomeadamente a realização de maior número de hemoculturas e a utilização de novas técnicas laboratoriais de biologia molecular (utilização de PCR para pneumococo desde 2008), permitindo a identificação de pneumococo que anteriormente não era detetado, nomeadamente nos líquidos pleurais e LCR de doentes já sob antibioterapia. Também constitui fator limitante da analise, o facto de não ter sido avaliado se o maior número de hemoculturas ocorreu em BO ou noutros diagnósticos.

Alguns centros dos EUA observaram haver menor realização de hemoculturas na BO após vacinação universal para pneumococo pela redução importante da incidência da doença. ${ }^{20}$ Entre nós essa redução não se verificou pelo facto da vacina não estar no PNV, tendo assim uma cobertura mais baixa e não uniforme. Simultaneamente, razões de vigilância epidemiológica de uma doença prevenível por vacinação, poderão também ter contribuído para tal. Os serotipos mais frequentemente isolados estão contidos na VCP13v (75,5\%; 37/49), destacando-se o 3 e 19A, correspondentes também a serotipos frequentemente isolados em Portugal. ${ }^{9}$ O pequeno número de serotipos disponíveis não permitiu analisar a sua evolução e a distribuição por grupos etários afetados. Fenoll $\mathrm{A}$ et al analisaram as tendências seculares (1990 - 2013) dos serotipos na era pré e pós-vacinal em regiões de Espanha sem vacinação universal. Demonstraram a ausência de impacto de baixas coberturas na incidência de doença causada por alguns serotipos vacinais, concluindo pela necessidade de esquemas universais de vacinação para maiores benefícios da mesma na DIP. ${ }^{21}$

Ocorreu um caso de DIP pelo serotipo vacinal 3 numa criança que tinha esquema vacinal adequado. Antachopoulos et al publicaram em 2014 uma série de derrames pleurais parapneumomónicos causados por S. pneumoniae serotipo 3. Cinco das 15 eram crianças que tinham recebido o esquema vacinal de VCP13v recomendado para a idade. ${ }^{22}$ Estudos serológicos prévios já tinham demonstrado níveis de anticorpos IgG contra o serotipo três mais baixos relativamente aos restantes serotipos vacinais em crianças vacinadas. ${ }^{23,24}$

\section{CONCLUSÃO}

Esta análise retrospetiva permitiu avaliar a evolução da DIP ao longo de 20 anos, antes e após licenciamento das vacinas conjugadas pneumocócicas e imediatamente antes da sua utilização universal em Portugal. Não houve uma tendência óbvia de redução dos casos de doença nesta população, mas, o período analisado é longo o que dificulta uma análise homogénea dos dados, nomeadamente pela introdução de novas técnicas laboratoriais de diagnóstico nos últimos anos, realização de mais hemoculturas e porque o número de casos é relativamente pequeno.

A introdução da VCP13v no PNV irá certamente contribuir para um melhor controlo da doença em Portugal e os dados aqui publicados serão importantes para avaliar a evolução da DIP em idade pediátrica e a repercussão noutros grupos etários pela imunidade de grupo.

\section{OBSERVAÇÕES}

Apresentado na forma de comunicação oral no $16^{\circ}$ Congresso Nacional de Pediatria, outubro 2015, Albufeira

\section{PROTECÇÃO DE PESSOAS E ANIMAIS}

Os autores declaram que os procedimentos seguidos estavam de acordo com os regulamentos estabelecidos pelos responsáveis da Comissão de Investigação Clínica e Ética e de acordo com a Declaração de Helsínquia da Associação Médica Mundial. 


\section{CONFIDENCIALIDADE DOS DADOS}

Os autores declaram ter seguido os protocolos do seu centro de trabalho acerca da publicação de dados.

\section{CONFLITOS DE INTERESSE}

Fernanda Rodrigues participou em advisory boards e foi palestrante em simpósios organizados pela GSK e Pfizer, com honorários pagos à Associação de Saúde Infantil de Coimbra (ASIC).

\section{REFERÊNCIAS}

1. Pilishvili T, Brendan N, Moore MR. Pneumococcal disease. In: VPD surveillance manual. $5^{\text {th }}$ ed. 2012:1-11. [consultado 2016 set 16]. Disponível em: https://www.cdc.gov/vaccines/pubs/surv-manual/chpt11pneumo.pdf.

2. Kaplan SL, Mason EO Jr, Wald ER, Schutze GE, Bradeley JS, Tan TQ, et al. Decrease of invasive pneumococal infections in children among 8 children's hospitals in the United States after the introduction of the 7-valent pneumococcal conjugate vaccine. Pediatrics. 2004;113:443-9.

3. De Lencastre H, Kristinsson KG, Brito-Avô A, Sanches IS, Sá-Leão R, Saldanha J, et al. Carriage of respiratory tract pathogens and molecular epidemiology of Streptococcus pneumoniae colonization in healthy children attending day care centers in Lisbon, Portugal. Microb Drug Resist.1999;5:19-29.

4. Rodrigues F, Foster D, Caramelo F, Serranho P, Gonçalves G, Januário $\mathrm{L}$, et al. Progressive changes in pneumococcal carriage in children attending daycare in Portugal after 6 years of gradual conjugate vaccine introduction show falls in most residual vaccine serotypes but no net replacement or trends in diversity. Vaccine. 2012;30:3951-6.

5. Recomendações sobre vacinas: actualização 2014. Comissão de Vacinas da Sociedade de Infecciologia Pediátrica e Sociedade Portuguesa de Pediatria. 2014: 13-18. [consultado 2016 set 16]. Disponível em: http://www.spp.pt/UserFiles/file/Comissao_de_Vacinas/ RECOMENDACOES_SOBRE_VACINAS_EXTRA_PNV_2014\%20 _1_\%20FINAL.pdf.

6. Tendais-Almeida $M$, Ferreira-Magalhães $M$, Alves I, Tavares $M$, Azevedo I. Vacinação contra infecções por Streptococcus pneumoniae em crianças e adolescentes de alto risco para doença invasiva pneumocócica. Acta Med Port. 2015;28:583-9.

7. Pilishvili T, Lexau C, Farley MM, Hadler J, Harrison LH, Bennett NM, et al. Sustained reductions in invasive pneumococcal disease in the era of conjugate vaccine. J Infect Dis. 2010;201:32-41.

8. Miller E, Andrews NJ, Waight PA, Slack MP, George RC. Herd immunity and serotype replacement 4 years after seven-valent pneumococcal conjugate vaccination in England and Wales: an observational cohort study. Lancet Infect Dis. 2011;11:760-8

9. Aguiar SI, Brito MJ, Horácio AN, Lopes JP, Ramirez M, Melo-Cristino J. Grupo de estudo da DIP da Secção de Infecciologia Pediátrica da SPP. Decreasing incidence and changes in seotype distribution of invasive pneumococcal disease in persons aged under 18 years since introduction of 10-valent and 13-valent conjugate vaccines in Portugal, July 2008 to June 2012. Euro Surveill. 2014;19:20750.

10. Recomendações sobre vacinas extra programa nacional de vacinação. Comissão de Vacinas da Sociedade de Infecciologia Pediátrica e Sociedade Portuguesa de Pediatria. [consultado 2016 set 16]. Disponível em: http://www.spp.pt/UserFiles/file/Comissao_de_Vacinas/ Recomendacoes\%20sobre\%20vacinas\%20extra\%20PN̄ $\%$ 2020152016.pdf.

11. Instituto Nacional de Estatística. Statistical information. Lisbon: INE. [consultado 2016 set 16]. Disponível em: http://www.ine.pt/

12. Moore MR, Whitney C. Use of pneumococcal disease epidemiology
AASIC recebeu da Pfizer apoio a projetos de investigação coordenados por Fernanda Rodrigues.

Os restantes autores declaram não terem conflitos para a elaboração do artigo

\section{FONTES DE FINANCIAMENTO}

Não existiram fontes externas de financiamento para realização deste artigo.

to set policy and prevent disease during 20 years of the emerging infections program. Emerg Infect Dis. 2015;21:1551-6.

13. Knol MJ, Wagenvoort GH, Sanders EA, Elberse K, Vlaminclx BJ, de Melker HE, et al. Invasive pneumococcal disease 3 years after introduction of 10-valent pneumococcal conjugate vaccine, the Netherlands. Emerg Infect Dis. 2015;21:2040-4.

14. Diawara I, Zerouali K, Katfy K, Zaki B, Belabbes H, Najib J, et al. Invasive pneumococcal disease among children younger than 5 years of age before and after introduction of pneumococcal conjugate vaccine in Casablanca, Morocco. Int J Infect Dis. 2015;40:95-101.

15. Verani JR, Domingues CM, de Moraes JC, Brazilian Pneumococcal Conjugate Vaccine Effectiveness Study Group. Indirect cohort analysis of 10 -valent pneumococcal conjugate vaccine effectiveness against vaccine-type and vaccine-related invasive pneumococcal disease. Vaccine. 2015;33:6145-8.

16. Varon E, Cohen R, Béchet S, Doit C, Levy C. Invasive disease potentia of pneumococci before and after the 13-valent pneumococcal conjugate vaccine implementation in children. Vaccine. 2015;33:6178-85.

17. SINAVE. Doenças de Notificação Obrigatória. Despacho 5687-A-2014 de 29 de Abril. [consultado 2016 set 16 ]. Disponível em: https://dre.pt/ application/dir/pdf2sdip/2014/04/082000001/0000200020.pdf.

18. Neves JF, Rodrigues P, Peres A, Constantino C, Cunha F. Doença pneumocócica invasiva na era vacinal. Acta Pediatr Port. 2008;39:206 10.

19. Pereira H, Preto C, Sousa M, Dias F, Gaspar E. Doença pneumocócica invasiva: treze anos de experiência num hospital de grupo II. Acta Pediatr Port. 2014;45:193-7.

20. Simon AE, Lukacs SL, Mendola P. National trends in emergency department use of urinalysis, complete blood count, and blood culture for fever without a source among children aged 2 to 24 months in the pneumococcal conjugate vaccine 7era. Pediatr Emerg Care. 2013;29:560-7.

21. Fenoll A, Granizo J, Giménez MJ, Yuste J, Aguilar L. Secular trends (1990-2013) in serotypes and associated non-susceptibility of S. pneumoniae isolates causing invasive disease in the pre-/post-era of pneumococcal conjugate vaccines in Spanish regions without universal paediatric pneumococcal vaccination. Vaccine. 2015;33:5691-9.

22. Antachopoulos C, Tsolia MN, Tzanakaki G, Xirogionni A, Dedous O, Markou G, et al. Parapneumonic pleural effusions caused by Streptococcus pneumoniae serotype 3 in children immunized with 13 -valent conjugated pneumococcal vaccine. Pediatr Infect Dis J. 2014; 33:81-3.

23. Yeh SH, Gurtman A, Hurley DC, Block SL, Schwartz RH, Patterson S, et al. Immunogenicity and safety of 13-valent pneumococcal conjugate vaccine in infants and toddlers. Pediatrics. 2010;126:e493-505.

24. Snape MD, Klinger CL, Daniels ED, John TM, Layton H, Rollinson L, et al. Immunogenicity and reactogenicity of a 13-valent-pneumococcal conjugate vaccine administered at 2,4 , and 12 months of age: a double-blind randomized active-controlled trial. Pediatr Infect Dis J. 2010;29:e80-90. 